\title{
On-chip light detection using monolithically integrated quantum dot micropillars
}

Cite as: Appl. Phys. Lett. 108, 081110 (2016); https://doi.org/10.1063/1.4942650

Submitted: 07 January 2016 . Accepted: 10 February 2016 . Published Online: 26 February 2016

M. M. Karow (D), P. Munnelly, T. Heindel (D), M. Kamp, S. Höfling, C. Schneider, and S. Reitzenstein (D)
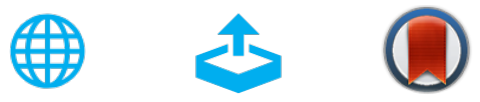

View Online

Export Citation

CrossMark

\section{ARTICLES YOU MAY BE INTERESTED IN}

Two-photon interference from remote deterministic quantum dot microlenses

Applied Physics Letters 110, 011104 (2017); https://doi.org/10.1063/1.4973504

Efficient single-photon source based on a deterministically fabricated single quantum dot microstructure with backside gold mirror

Applied Physics Letters 111, 011106 (2017); https://doi.org/10.1063/1.4991389

An electrically driven cavity-enhanced source of indistinguishable photons with $61 \%$ overall efficiency

APL Photonics 1, 011301 (2016); https://doi.org/10.1063/1.4939831

\section{Lock-in Amplifiers up to $600 \mathrm{MHz}$}
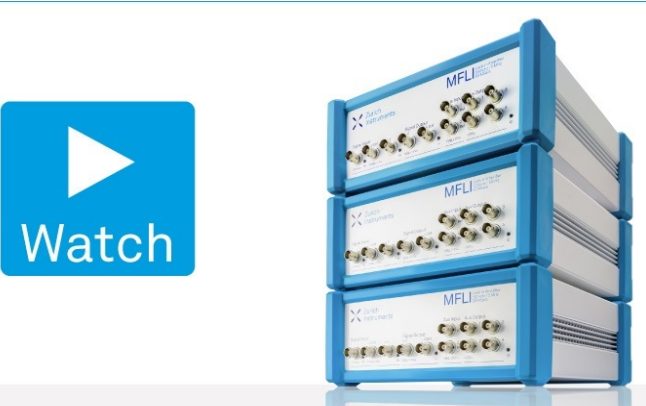


\title{
On-chip light detection using monolithically integrated quantum dot micropillars
}

\author{
M. M. Karow, ${ }^{1, a)}$ P. Munnelly, ${ }^{1}$ T. Heindel, ${ }^{1, b)}$ M. Kamp, ${ }^{2}$ S. Höfling, ${ }^{2,3}$ C. Schneider, ${ }^{2}$ \\ and S. Reitzenstein ${ }^{1}$ \\ ${ }^{1}$ Institut für Festkörperphysik, Technische Universität Berlin, 10623 Berlin, Germany \\ ${ }^{2}$ Technische Physik, Universität Würzburg, 97074 Würzburg, Germany \\ ${ }^{3}$ SUPA, School of Physics and Astronomy, University of St Andrews, St Andrews KY16 9SS, United Kingdom
}

(Received 7 January 2016; accepted 10 February 2016; published online 26 February 2016)

\begin{abstract}
We demonstrate the on-chip detection of light using photosensitive detectors based on quantum dot micropillar cavities. These microscale detectors are applied exemplarily to probe the emission of a monolithically integrated, electrically pumped whispering gallery mode microlaser. Light is detected via the photocurrent induced in the electrically contacted micropillar detectors under reverse-bias. In order to demonstrate the high potential and applicability of the microdetector presented, we determine the threshold current of an integrated microlaser to be $(54 \pm 4) \mu \mathrm{A}$, in very good agreement with the value of $(53 \pm 4) \mu \mathrm{A}$ inferred from the optical data. Within this work, we realize the monolithic integration of a laser and a detector in a single device operating in the regime of cavity-quantum electrodynamics. Our results thus advance the research on microscale sensor technology towards the few-photon quantum limit and pave the way for on-chip opto-electronic feedback experiments. (C) 2016 AIP Publishing LLC. [http://dx.doi.org/10.1063/1.4942650]
\end{abstract}

Integrated light sources $^{1,2}$ and detectors ${ }^{3,4}$ have high potential to play a key role in future photonic devices. In particular, they can act as compact, low-power building blocks in optical communication and information technologies. ${ }^{5-7}$ Within these fields, semiconductor quantum dots (QDs) and QD-microcavity systems show great promise for advancing integrated photonics not only in terms of light sources but also with regard to detection. In fact, single-photon detection, crucial to quantum information processing, has been achieved using QDs in transistor-, ${ }^{8}$ resonant-tunnelling diode--, ${ }^{9}$ and single-QD-based devices. ${ }^{10}$ Integrating the QDs within a resonator further improves the light detection efficiency as well as the wavelength selectivity ${ }^{11}$ and advances the research in the single-emitter regime of cavity-quantum electrodynamics (cQED). ${ }^{12}$ Regarding the generation of light, micropillar cavities with embedded QDs are particularly viable as integrated, electrically operated light sources as they exhibit high $Q$-factors and low mode volumes. As such, they provide cQEDenhanced light-matter interaction, ${ }^{13-15}$ which allows for the realization of low-threshold high- $\beta$ lasers for both vertically propagating modes ${ }^{16}$ and laterally propagating whispering gallery modes (WGMs). ${ }^{17,18}$ Further, due to their laterally directed emission, WGM microlasers are of special interest in the context of coherent light sources for on-chip quantum optical studies $^{19}$ and the generation of non-classical light, ${ }^{20}$ as well as for driving optical circuits due to their compatibility with planar photonic networks. $^{21-23}$ To this end, integrating such lasers with microscale detectors opens up interesting perspectives, such as wavelength-selective microscale sensing applications and opto-electronic feedback experiments. The latter can lead

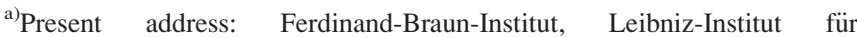
Höchstfrequenztechnik, Gustav-Kirchhoff-Str. 4, 12489 Berlin, Germany. Electronic mail: matthias.karow@fbh-berlin.de.

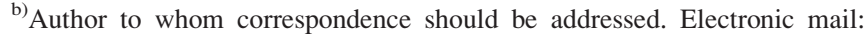
tobias.heindel@tu-berlin.de
}

to chaotic optical and electrical output behavior enabling, e.g., compact and ultrafast random number generators. ${ }^{24,25}$ In this view, the integration of laterally coupled laser-detector pairs is highly desirable as it allows for the realization of such optoelectronic feedback ensembles on a single chip.

In this letter, we report on the integration of a microscale photodetector into a cQED system. The QD-based onchip microdetector is applied to characterize the emission from a monolithically integrated WGM microlaser via electrical readout of the photocurrent. In this configuration, we go a significant step beyond the conventional approach in which individual micropillars have been used as photosensitive devices under axial light incoupling. ${ }^{12}$ In order to demonstrate the potential of our approach, we use a differential lock-in technique to probe the input-output curve of the integrated microlaser and to determine its threshold current.

A schematic of the integrated microlaser-photodetector pair is shown in Fig. 1(a). The sample is based on a planar microcavity structure grown by molecular beam epitaxy on an n-doped (100)-oriented GaAs substrate. Therein, n- and p-doped distributed Bragg reflectors with 27 and $23 \lambda / 4 \mathrm{AlAs} / \mathrm{GaAs}$ mirror pairs are grown below and above an intrinsic GaAs $\lambda$-cavity, respectively. A single layer of self-organized InGaAs QDs (area density $\sim 10^{9} \mathrm{~cm}^{-2}$ ) treated with partial capping and annealing processes $^{26}$ serves as gain medium located within the $\lambda$-cavity. From this wafer, micropillars with a diameter of $4 \mu \mathrm{m}$ serving as on-chip coherent light sources (via lasing WGM emission) were patterned by electron-beam lithography and reactive ion plasma etching. Surrounding the characterized microlaser is a group of five $1.6 \mu \mathrm{m}$-diameter peripheral micropillars, separated from the WGM microlaser by a distance of $15 \mu \mathrm{m}$, which act as monolithically integrated photodetectors (cf. inset, Fig. 1(a)). A planarization step with the polymer bencocyclobutene was followed by the application of Au pads serving as electrical contacts, with separate p-contacts for the laser bar and the detector bar. ${ }^{27}$ 


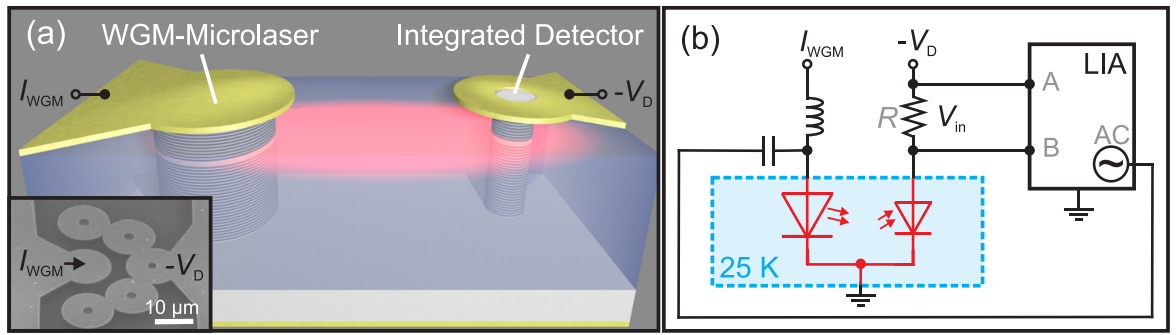

FIG. 1. Device layout and electrical circuit used for the measurement of photocurrent from an integrated photodetector. (a) Schematic of the device configuration featuring a whispering gallery mode (WGM) microlaser, which, upon electrical excitation, generates photocarriers in a monolithically integrated peripheral micropillar used as on-chip photodetector. The inset shows a scanning electron micrograph of an exemplary device with the WGM laser and its neighboring photodetectors. (b) This circuit is used to determine the microlaser's threshold current in a photocurrent extraction experiment in which the biased laser $\left(I_{\mathrm{WGM}}\right)$ is modulated by a small sinusoidal $(\mathrm{AC})$ signal for detection via a lock-in amplifier (LIA).

The device was mounted on a chip carrier within a liquid-Helium flow cryostat and cooled to $25 \mathrm{~K}$ for electrical and optical characterization. The former was carried out with a source-measurement unit for direct current (DC) injection/ bias application and an optional lock-in amplifier (LIA) providing a sinusoidal reference voltage output. The equivalent circuit diagram of the experiment can be seen in Fig. 1(b). The optical characterization was carried out in a microelectroluminescence ( $\mu \mathrm{EL})$ setup featuring a $20 \times$ microscope objective (numerical aperture 0.42), a triple-grating monochromator with a spectral resolution of $85 \mu \mathrm{eV}$, and a liquid-nitrogen cooled charge-coupled device camera.

To compare and validate the results obtained from our on-chip detector concept, the WGM microlaser's emission was first characterized in a conventional $\mu$ EL setup to optically determine the input-output characteristic and the threshold current in off-chip configuration. For this purpose, the WGM laser emission scattered at marker structures located in the vicinity of the microlaser was collected via the microscope objective, and spectra were recorded for injection currents up to $312 \mu \mathrm{A}$ (see Fig. 2(a)). The microlaser was found to provide single-mode emission at $852.6 \mathrm{~nm}$ with a $Q$-factor of 13500 at threshold. The lasing mode was fitted in each case with a Voigt function, taking into account the spectral resolution of the setup, from which the full-width at half-maximum (FWHM) and integrated intensity were extracted. As seen in Fig. 2(b), at low injection currents, the WGM mode has a linewidth of $250 \mu \mathrm{eV}$. This is followed by a sudden decrease down to the resolution limit of the setup $(85 \mu \mathrm{eV})$ at elevated currents, indicating the onset of lasing.
Likewise, the input-output curve reveals a smooth transition into linear scaling around threshold. Its value was determined to be $I_{\text {th }}=(53 \pm 4) \mu \mathrm{A}$ (at $6.2 \mathrm{~V}$ laser bias).

Reverse-biasing the peripheral micropillars allows one to electrically probe the microlaser emission. Once the WGM microlaser is turned on, the reverse current in the peripheral micropillars increases due to the generation of photocarriers within the active region. Photocurrent generation was also verified for each microdetector by individual excitation with an external tunable laser (not shown). Fig. 3(a) depicts $I-V$ curves (black) of the peripheral micropillars at no laser current (dashed) and at $312 \mu \mathrm{A}$ (solid) from a direct measurement of the current flowing (without LIA). At vanishing laser current, the moderately reverse-biased detectors show a dark current below $10 \mathrm{nA}$, while at about $-10 \mathrm{~V}$, the junction reaches breakdown. Upon laser emission, the reverse current increases by the amount of the extracted photocurrent (blue). Its value increases as the detector voltage is varied from 1.4 (flatband condition) to $-9 \mathrm{~V}$, since injected photocarriers are extracted with increasing efficiency from the QD ensemble due to enhanced tunneling processes. ${ }^{28}$ At detector voltages below $-9 \mathrm{~V}$, photocarriers are multiplied by impact ionization, as apparent from the sudden increase in photocurrent. The breakdown voltage of the junction shifts slightly to $-9 \mathrm{~V}$, as a result of the additional impact-ionized photocarriers. This direct operation without the lock-in technique allowed a linear response to the optical emission (Fig. 2(a)) to be verified, see Fig. 3(b). The responsivity was found to be about $0.05 \mathrm{~A} / \mathrm{W}$ for biases up to $-8 \mathrm{~V}$. Taking into account the dark current at this working point, this corresponds to a noise-equivalent
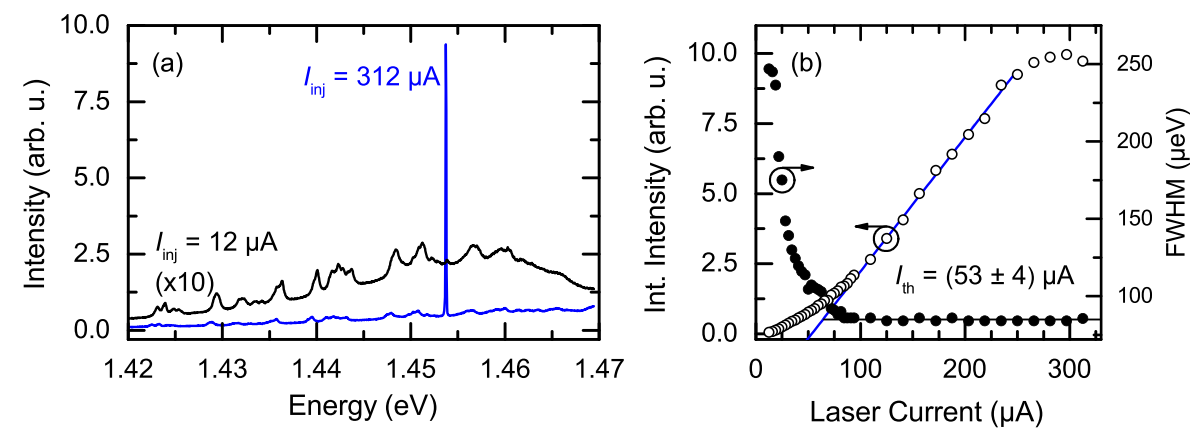

FIG. 2. (a) Two exemplary spectra of the WGM microlaser at an injection current $I_{\text {inj }}$ of 12 and $312 \mu \mathrm{A}$ corresponding to a laser bias of 3.5 and $12.1 \mathrm{~V}$, respectively. (b) Optical characteristic of this $4 \mu$ m-diameter laser. A sudden decrease of the FWHM down to the resolution limit of the setup is accompanied by a transition of the integrated intensity into a linear dependence. The threshold current of $(53 \pm 4) \mu \mathrm{A}$ was deduced from the linear fit (solid straight line). 

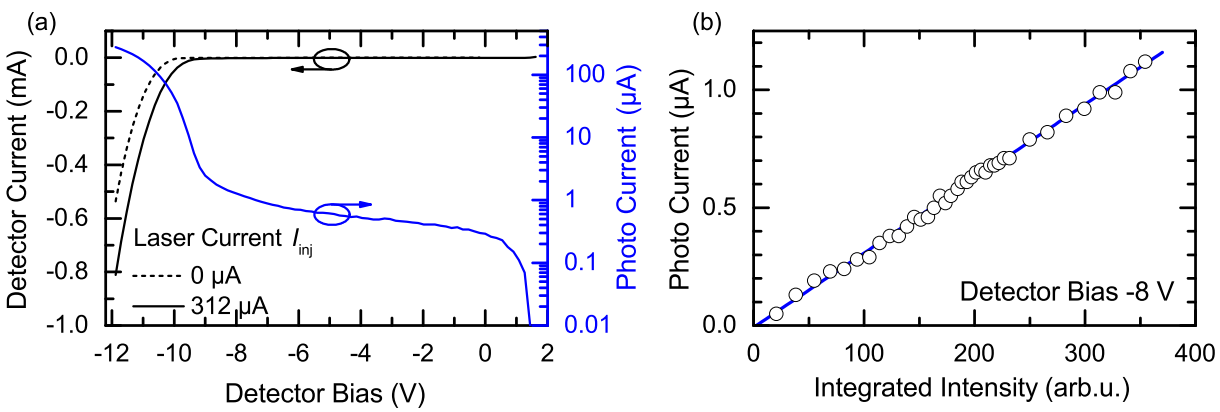

FIG. 3. (a) $I-V$ curves (black) of the peripheral micropillars at no laser current (dashed) and at a laser current of $312 \mu \mathrm{A}$ (solid). The photocurrent at this laser current (blue) is calculated as the difference between the two $I-V$ curves. Photocarriers injected by the WGM laser led to an increased detector current, and the former is extracted more efficiently from the QD ensemble at higher reverse biases, until junction breakdown at $-9 \mathrm{~V}$ initiates strong carrier multiplication. (b) The photodetector exhibits a linear response to the optical input power generated by the integrated WGM microlaser. The integrated intensity is deduced from $\mu$ EL spectra exemplarily shown in Fig. 2(a).

power (NEP) of $6.3 \times 10^{-9} \mathrm{~W} / \mathrm{Hz}^{1 / 2}$. Further, by considering the effective detector area of the five coupled micropillar detectors, we gain an upper limit for the specific detectivity of $D^{*}=1 \times 10^{5} \mathrm{~cm} \mathrm{~Hz}^{1 / 2} / \mathrm{W}$. This rather moderate value is explained by the fact that our detectors are based on a single low-density layer of InGaAs QDs (areal density $\sim 10^{9} \mathrm{~cm}^{-2}$ ) in the active layer. We expect that $D^{*}$ can be significantly enhanced by 2-3 orders of magnitude via stacked layers (e.g., 10 layers) of QDs with high areal density $\left(10^{10}-10^{11}\right) \mathrm{cm}^{-2}$ in the active region, which would result in a performance comparable to reports on $\mathrm{QD}^{29}$ and quantum well ${ }^{30}$-based photodetectors utilizing nanophotonic approaches. Moreover, WGM microlasers with Limaçon-shaped cross-section, providing in-plane unidirectional emission into two light cones, ${ }^{21}$ can strongly enhance the overall coupling efficiency of the integrated microlaser-microdetector system.

After demonstrating the capability of our highly integrated approach for on-chip photocurrent extraction, we apply this appealing feature exemplarily to infer the threshold current of the WGM microlaser being a crucial parameter of coherent light sources. For this purpose, the circuit shown in Fig. 1(b) is utilized. The LIA allows us to apply a differential acquisition method which is particularly sensitive to a time-varying lasing mode intensity, as elaborated in the following. It can be inferred from the right branch of the circuit that the input voltage $V_{\text {in }}$ to the LIA is given by $V_{\text {in }}(\mathrm{t})=V_{\mathrm{D}} /\left(1+R_{\mathrm{D}}(\mathrm{t}) / R\right)$, as derived from Kirchhoff's law. Here, the detector voltage $V_{\mathrm{D}}$, its resistance $R_{\mathrm{D}}$, and the readout resistance $R$ are introduced. If a small $\mathrm{AC}$ modulation with a frequency $\omega$ is added to the constant current $I_{\mathrm{WGM}}$ (left branch Fig. 1(b)), $R_{\mathrm{D}}$ is reduced according to $R_{\mathrm{D}}(\mathrm{t})=R_{0}-R_{\mathrm{PC}}^{\mathrm{DC}}-R_{\mathrm{PC}}^{\mathrm{AC}} \times \sin (\omega \mathrm{t})$, as a result of absorption of an optical power $\mathrm{P}_{\mathrm{opt}}$. We introduced the diode's resistance $R_{0}$ under no illumination and appropriate resistance quantities $R_{\mathrm{PC}}^{\mathrm{DC}}$ and $R_{\mathrm{PC}}^{\mathrm{AC}}$ accounting for diode resistancelowering upon injection. The time-varying input signal $V_{\text {in }}(\mathrm{t})$ is processed by the LIA to the readout voltage $V_{\mathrm{LIA}}=(1 / 2 \sqrt{2})$ $\times\left(\max _{\mathrm{t}}\left(V_{\text {in }}\right)-\min _{\mathrm{t}}\left(V_{\text {in }}\right)\right)$. Approximating this expression in the limit $R_{\mathrm{PC}}^{\mathrm{DC}} / R \ll 1$ and $R_{\mathrm{PC}}^{\mathrm{AC}} / \mathrm{R} \ll 1$ yields

$$
\begin{aligned}
V_{\mathrm{LIA}}\left(I_{\mathrm{WGM}}\right)= & \left(\alpha V_{\mathrm{D}} / \sqrt{2}\right) \times \Delta P_{\mathrm{opt}}\left(I_{\mathrm{WGM}}\right) \\
& \times\left(\frac{\alpha \times P_{\mathrm{opt}}\left(I_{\mathrm{WGM}}\right)}{\left(1+R_{0} / R\right)^{3}}+\frac{1}{\left(1+R_{0} / R\right)^{2}}\right) .
\end{aligned}
$$

Above threshold $\left(I_{\mathrm{WGM}}>I_{\mathrm{th}}\right)$, Eq. (1) describes a function linear in $I_{\mathrm{WGM}}$ since $P_{\mathrm{opt}}$ is a linear function of $I_{\mathrm{WGM}}$ and, hence, $\Delta P_{\mathrm{opt}}\left(I_{\mathrm{WGM}}>I_{\mathrm{th}}\right)=$ const. Upon subtraction of this superimposed linear trend in the limit $R_{0} / R \gg 1$, one obtains the pseudo-LIA voltage $V_{\mathrm{LIA}}^{\dagger}\left(I_{\mathrm{WGM}}\right) \propto V_{\mathrm{D}} \times\left(\Delta P_{\text {opt }}\left(I_{\mathrm{WGM}}\right)-\right.$ $\left.\Delta P_{\text {opt }}\left(I_{\mathrm{WGM}}>I_{\mathrm{th}}\right)\right)$. As the microlaser's input-output curve $P_{\mathrm{opt}}\left(I_{\mathrm{WGM}}\right)$ can be derived from

$V_{\mathrm{LIA}}^{\dagger}\left(I_{\mathrm{WGM}}\right)$ by integration

$$
P_{\mathrm{LIA}}=\int_{0}^{I_{\mathrm{WGM}}} V_{\mathrm{LIA}}^{\dagger}\left(I_{\mathrm{WGM}}^{\prime}\right) \mathrm{d} I_{\mathrm{WGM}}^{\prime} \propto P_{\mathrm{opt}}\left(I_{\mathrm{WGM}}\right)+C,
$$

the value of the laser threshold current is directly accessible.

In Fig. 4, we display experimental data obtained in two threshold-determination experiments with two exemplary detector bias voltages $V_{\mathrm{D}}$ of -4 and $-8 \mathrm{~V}$, respectively (500 $\Omega$ readout resistance $R$ ) over a range of laser currents between 8 and $160 \mu \mathrm{A}$ ( 3 and $9.5 \mathrm{~V}$ laser bias, respectively). The sinusoidal amplitude for the small signal modulation was chosen as $0.1 \mathrm{~V}$ at a frequency of $102 \mathrm{kHz}$. The LIA readout $V_{\text {LIA }}$ which varies with laser current can be inspected in Fig. 4(a). As described previously (cf. Eq. (1)), $V_{\text {LIA }}$ scales linearly with $I_{\mathrm{WGM}}$ at high currents. At lower laser currents, the data show a transition into a linear trend with a clear kink at around $50 \mu \mathrm{A}$. This is illustrated by the pseudo-LIA voltage $V_{\text {LIA }}^{\dagger}$ shown in Fig. 4(b) for which this superimposed linear function was subtracted from $V_{\text {LIA }}$. It closely resembles the derivative of the microlaser's input-output curve. Numerical integration of $V_{\mathrm{LIA}}^{\dagger}$ (cf. Eq. (2)) retrieves the microlaser's input-output curve as shown in Fig. 4(c). Linear fits to the high-current branches allow for an extraction of the laser threshold current defined as the abscissa intercept with the linear functions. In order to verify the result, this experiment was carried out in more than ten different configurations $\left(R \in[75: 10 \mathrm{k}] \Omega\right.$ and $\left.V_{\mathrm{D}} \in[-8: 0] \mathrm{V}\right)$ yielding the threshold current $(54 \pm 4) \mu \mathrm{A}$.

In summary, we have demonstrated the realization of onchip microscale photodetection and its application to characterize a monolithically integrated cQED-enhanced device. Using such integrated microdetectors, we all-electrically determined the threshold current of a WGM microlaser via a lock-in technique. The results presented in this report show promise for the exploration of self-pulsing and chaos for, e.g., random number generation and chaos-based secure 


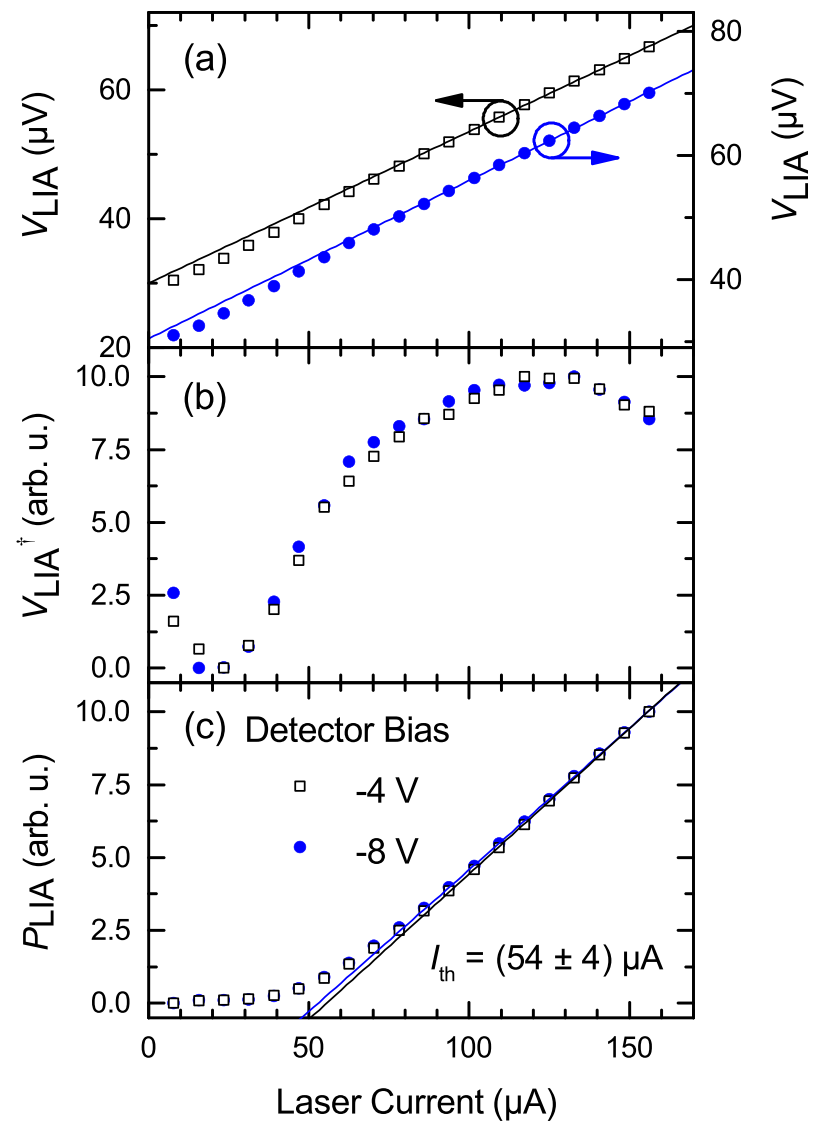

FIG. 4. Experimental data for all-electrical threshold-current determination with a phase-sensitive method. Two different configurations with a detector bias of -4 (black squares) and $-8 \mathrm{~V}$ (blue circles) are used. (a) LIA-output voltage, originating from the time-varying voltage drop across a resistor in series with the photodetector, against the laser current. Linear curves are fitted to the upper branches (laser current greater than $90 \mu \mathrm{A}$ ). (b) Subtracting the linear trend superimposed to $V_{\text {LIA }}$ yields this curve, which closely resembles the derivative of the laser's input-output curve (normalized). (c) Numerical integration (cf. Eq. (2)) gives the input-output curve $P_{\text {LIA }}$ (normalized), from which the threshold current can be extracted.

communication, as it is possible to read out the laser-induced photocurrent and use it to modulate the current flowing through the microlaser in an on-chip feedback-type experiment. Beyond this, the presented concept of a monolithically integrated microlaser-photodetector pair might be utilized for on-chip chemical sensing applications. To realize such microscale absorption spectroscopy, WGM lasers emitting in the near-infrared ${ }^{31}$ and operating at room temperature ${ }^{32}$ can be exploited in future.

This work was supported by the German Research Foundation (DFG) under Grant Nos. RE2974/9-1 and SCHN1376/1-1. The research leading to these results has received funding from the European Research Council under the European Union's Seventh Framework ERC Grant Agreement No. 615613. The authors gratefully thank M. Lermer, M. Emmerling, and A. Wolf for expert sample preparation.
${ }^{1}$ D. Englund, H. Altug, and J. Vučković, Appl. Phys. Lett. 91, 071124 (2007).

${ }^{2}$ T. B. Hoang, J. Beetz, M. Lermer, L. Midolo, M. Kamp, S. Höfling, and A. Fiore, Opt. Express 20, 21758 (2012).

${ }^{3}$ J. P. Sprengers, A. Gaggero, D. Sahin, S. Jahanmirinejad, G. Frucci, F. Mattioli, R. Leoni, J. Beetz, M. Lermer, M. Kamp, S. Höfling, R. Sanjines, and A. Fiore, Appl. Phys. Lett. 99, 181110 (2011).

${ }^{4}$ G. Reithmaier, S. Lichtmannecker, T. Reichert, P. Hasch, K. Müller, M. Bichler, R. Gross, and J. J. Finley, Sci. Rep. 3, 1901 (2013).

${ }^{5}$ M. Haurylau, G. Chen, H. Chen, J. Zhang, N. A. Nelson, D. H. Albonesi, E. G. Friedman, and P. M. Fauchet, IEEE J. Sel. Top. Quantum Electron. 12, 1699-1705 (2006).

${ }^{6}$ A. Shacham, K. Bergman, and L. P. Carloni, IEEE Trans. Comput. 57, 1246 (2008).

${ }^{7}$ L. Thylén and L. Wosinski, Photonics Res. 2, 75-81 (2014).

${ }^{8}$ A. J. Shields, M. P. O’Sullivan, I. Farrer, D. A. Ritchie, R. A. Hogg, M. L. Leadbeater, C. E. Norman, and M. Pepper, Appl. Phys. Lett. 76, 3673 (2000).

${ }^{9}$ J. C. Blakesley, P. See, A. J. Shields, B. E. Kardynał, P. Atkinson, I. Farrer, and D. A. Ritchie, Phys. Rev. Lett. 94, 067401 (2005).

${ }^{10}$ S. Komiyama, O. Astafiev, V. Antonov, T. Kutsuwa, and H. Hirai, Nature 403, 405 (2000).

${ }^{11}$ C. Kistner, S. Reitzenstein, C. Schneider, S. Höfling, and A. Forchel, Appl. Phys. Lett. 94, 221103 (2009).

${ }^{12}$ P. Gold, M. Gschrey, C. Schneider, S. Höfling, A. Forchel, M. Kamp, and S. Reitzenstein, Phys. Rev. B 86, 161301(R) (2012).

${ }^{13}$ J. M. Gérard, B. Sermage, B. Gayral, B. Legrand, E. Costard, and V. Thierry-Mieg, Phys. Rev. Lett. 81, 1110 (1998).

${ }^{14}$ K. J. Vahala, Nature 424, 839 (2003).

${ }^{15}$ J. P. Reithmaier, G. Sek, A. Löffler, C. Hofmann, S. Kuhn, S. Reitzenstein, L. V. Keldysh, V. D. Kulakovskii, T. L. Reinecke, and A. Forchel, Nature 432, 197 (2004).

${ }^{16}$ S. Reitzenstein, A. Bazhenov, A. Gorbunov, C. Hofmann, S. Münch, A. Löffler, M. Kamp, J. P. Reithmaier, V. D. Kulakovskii, and A. Forchel, Appl. Phys. Lett. 89, 051107 (2006).

${ }^{17}$ V. N. Astratov, S. Yang, S. Lam, B. D. Jones, D. Sanvitto, D. M. Whittaker, A. M. Fox, M. S. Skolnick, A. Tahraoui, P. W. Fry, and M. Hopkinson, Appl. Phys. Lett. 91, 071115 (2007).

${ }^{18}$ Y.-R. Nowicki-Bringuier, J. Claudon, C. Böckler, S. Reitzenstein, M. Kamp, A. Morand, A. Forchel, and J. M. Gérard, Opt. Express 15, 17291 (2007).

${ }^{19}$ E. Stock, F. Albert, C. Hopfmann, M. Lermer, C. Schneider, S. Höfling, A. Forchel, M. Kamp, and S. Reitzenstein, Adv. Mater. 25, 707 (2013).

${ }^{20}$ P. Munnelly, T. Heindel, M. M. Karow, S. Höfling, M. Kamp, C. Schneider, and S. Reitzenstein, IEEE J. Sel. Top. Quantum Electron. 21, 1900609 (2015).

${ }^{21}$ F. Albert, C. Hopfmann, A. Eberspächer, F. Arnold, M. Emmerling, C. Schneider, S. Höfling, A. Forchel, M. Kamp, J. Wiersig, and S. Reitzenstein, Appl. Phys. Lett. 101, 021116 (2012).

${ }^{22}$ A. W. Schell, J. Kaschke, J. Fischer, R. Henze, J. Wolters, M. Wegener, and O. Benson, Sci. Rep. 3, 1577 (2013).

${ }^{23}$ P. S. Kuo, J. Bravo-Abad, and G. S. Solomon, Nat. Commun. 5, 3109 (2014).

${ }^{24}$ I. Kanter, Y. Aviad, I. Reidler, E. Cohen, and M. Rosenbluh, Nat. Photonics 4, 58 (2010).

${ }^{25}$ M. Sciamanna and K. A. Shore, Nat. Photonics 9, 151 (2015).

${ }^{26}$ J. M. García, T. Mankad, P. O. Holtz, P. J. Wellman, and P. M. Petroff, Appl. Phys. Lett. 72, 3172 (1998).

${ }^{27}$ C. Böckler, S. Reitzenstein, C. Kistner, R. Debusmann, A. Löffler, T. Kida, S. Höfling, A. Forchel, L. Grenouillet, J. Claudon, and J. M. Gérard, Appl. Phys. Lett. 92, 091107 (2008).

${ }^{28}$ F. Hofbauer, S. Grimminger, J. Angele, G. Böhm, R. Meyer, M. C. Amann, and J. J. Finley, Appl. Phys. Lett. 91, 201111 (2007).

${ }^{29}$ S. C. Lee, S. Krishna, and S. R. J. Breuck, Opt. Express 17, 23160 (2009).

${ }^{30}$ S. Kalchmair, R. Gansch, S. I. Ahn, A. M. Andrews, H. Detz, T. Zederbauer, E. Mujagić, P. Reininger, G. Lasser, W. Schrenk, and G. Strasser, Opt. Express 20, 5622 (2012).

${ }^{31}$ A. Schlehahn, F. Albert, C. Schneider, S. Höfling, S. Reitzenstein, J. Wiersig, and M. Kamp, Opt. Express 21, 15951 (2013).

${ }^{32}$ M. Munsch, J. Claudon, N. S. Malik, K. Gilbert, P. Grosse, J. M. Gérard, F. Albert, F. Langer, T. Schlereth, M. M. Pieczarka, S. Höfling, M. Kamp, A. Forchel, and S. Reitzenstein, Appl. Phys. Lett. 100, 031111 (2012). 